\title{
Two-body problem of core-region coupled magnetic vortex stacks
}

\author{
Max Hänze, ${ }^{1, *}$ Christian F. Adolff, ${ }^{1}$ Sven Velten, ${ }^{1}$ Markus Weigand, ${ }^{2}$ and Guido Meier ${ }^{3,4}$ \\ ${ }^{1}$ Institut für Angewandte Physik und Zentrum für Mikrostrukturforschung, Universität Hamburg, 20355 Hamburg, Germany \\ ${ }^{2}$ Max-Planck-Institute for Intelligent Systems, Stuttgart, Germany \\ ${ }^{3}$ The Hamburg Centre for Ultrafast Imaging, Luruper Chaussee 149, 22761 Hamburg, Germany \\ ${ }^{4}$ Max-Planck Institute for the Structure and Dynamics of Matter, Luruper Chaussee 149, 22761 Hamburg, Germany
}

(Received 4 August 2015; revised manuscript received 26 January 2016; published 9 February 2016)

\begin{abstract}
The dynamics of all four combinations of possible polarity and circularity states in a stack of two vortices is investigated by time-resolved scanning transmission $\mathrm{x}$-ray microscopy. The vortex stacks are excited by unidirectional magnetic fields leading to a collective oscillation. Four different modes are observed that depend on the relative polarizations and circularities of the stacks. They are excited to a driven oscillation. We observe a repulsive and attractive interaction of the vortex cores depending on their relative polarizations. The nonlinearity of this core interaction results in different trajectories that describe a two-body problem.
\end{abstract}

DOI: 10.1103/PhysRevB.93.054411

\section{INTRODUCTION}

Magnetic structures found in thin ferromagnetic layers [1,2], such as magnetic bubbles [3], domain walls [4], skyrmions [5], and vortices [6], have been studied intensively over the past few decades. Their characteristic magnetization structures result from the minimization of different energy contributions. For instance, the formation of magnetic bubbles originates from a uniaxial anisotropy determined by the ferromagnetic layer. Magnetic skyrmions are commonly stabilized in the presence of external magnetic fields and an asymmetry in the boundary layers that leads to the Dzyaloshinskii-Moriya interaction [5]. Magnetic vortices emerge when the geometry of the ferromagnet is confined to dimensions on the micrometer scale [7], e.g., in micron-sized disks. Here, stray fields at the edges of the structures are minimized. The magnetic vortex constitutes a magnetization circulating in the plane around the center position of the disk where it points out of plane. The sense of circulation $c$ can be either clockwise or counterclockwise ( $c= \pm 1$ ), whereas the out-of-plane component of the center region points either up or down (polarization $p=$ \pm 1 ). Due to the four possible states, vortices are promising candidates for applications in potential storage devices [8,9]. In order to realize a storage device with a high storage density one needs to incorporate many vortices in a finite volume. Since neighboring vortices couple due to stray fields emerging at the surfaces of the ferromagnetic elements [10], the motions of closely packed vortices are strongly influenced by their surrounding ferromagnetic structures $[11,12]$. The interaction between laterally arranged elements has been studied for pairs [13,14], chains [15], and two-dimensional arrangements [16-18] of vortices. In laterally coupled arrangements it has been shown recently that memorylike writing processes are possible based on the excitation of the gyrotropic mode [19], where bits are stored as polarization patterns. The gyrotropic mode corresponds to a gyration of the vortex core around the center of the disk and can be compared to the oscillation of a harmonic oscillator [20]. Even for closely packed twodimensional arrays of vortices, the storage density is expected

\footnotetext{
*max.haenze@physnet.uni-hamburg.de
}

to be below that of conventional storage devices [21]. We introduce an additional dimension to the collective gyrations of vortices known from spin-torque oscillators [22]. Stacking the vortices allows for a strongly increased packing density and has thus stimulated recent studies [23-30]. While for two-dimensional arrangements the minimization of the stray fields at the side surfaces creates the vortices and mediates their interaction, we observe a second coupling mechanism for three-dimensional stacks that has been investigated theoretically $[26,31,32]$. Due to core coupling the collective motions in an elementary stack of two vortices become a two-body problem.

Here, we study the vortex core motions emerging in a stack of magnetic vortices depicted in Fig. 1 using time-resolved scanning transmission $\mathrm{x}$-ray microscopy. In the first step we excite the vortices by a short magnetic field pulse leading to a collective motion of the core regions. Both vortex cores are imaged along their damped oscillation. We observe a strong dependence of the resonance frequency and the trajectories of the gyration on the relative circulations and polarities within the stack. In the second step we excite the different nondegenerate states close to their resonance frequency. Stationary trajectories are observed that are comparable to motions of the gravitational two-body problem. This effect originates in the proximity of the vortex core regions within the stack. Calculations elucidate their functional dependence.

\section{SAMPLE PREPARATION AND METHODS}

Figure 1(a) depicts schematics of the measurement setup and $\mathrm{x}$-ray measurements of the investigated stack of vortices. Magnetic contrast is provided via the $\mathrm{X}$-ray magnetic circular dichroism (XMCD) at the $\mathrm{Ni} L_{3}$-absorption edge $(852.7 \mathrm{eV})$. The spatial resolution with the zone plate used in the present experiment is $25 \mathrm{~nm}$. The maximum temporal resolution is 40 ps. Stacks of polycrystalline permalloy $\left(\mathrm{Ni}_{80} \mathrm{Fe}_{20}\right)$ disks separated by an interlayer of silicon are prepared with electron-beam lithography, in situ thermal evaporation of permalloy/silicon/permalloy layers, and liftoff processing on a 100-nm-thick silicon nitride membrane. The disks have a diameter of $1 \mu \mathrm{m}$ and a thickness of $40 \mathrm{~nm}$. The silicon spacer has a thickness of $20 \mathrm{~nm}$. A coplanar waveguide is 
(a)

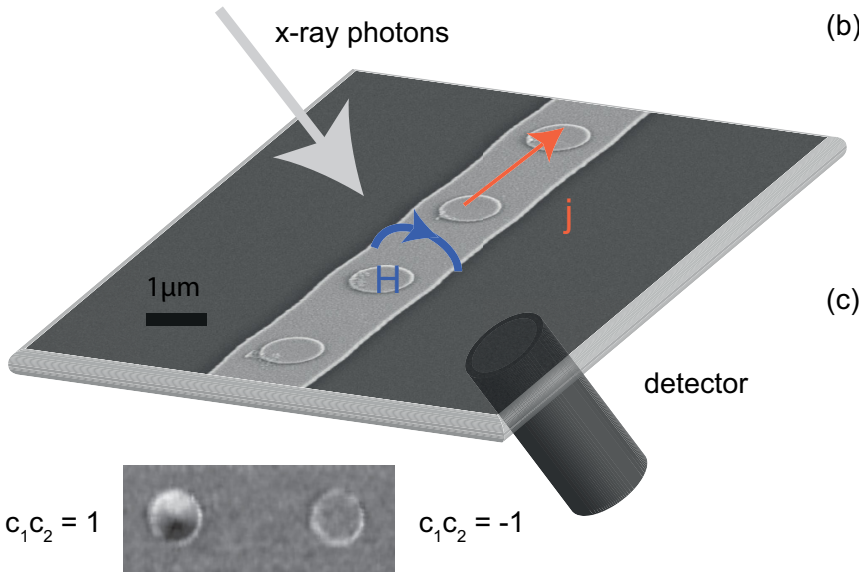

(b)

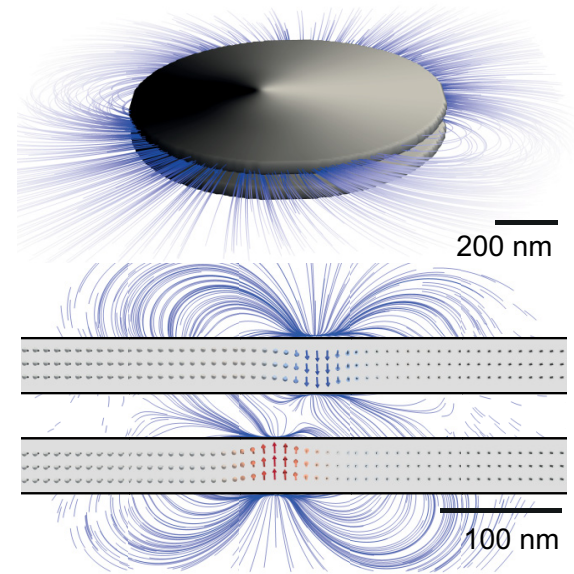

FIG. 1. (a) Schematics of the measurement setup along with scanning electron micrographs of the investigated ferromagnetic microstructures. The subset depicts micrographs of the vortex stacks with in-plane magnetic contrast using scanning transmission x-ray microscopy. The stray fields emerging (b) at the side surfaces and (c) the center regions of the ferromagnets are obtained from micromagnetic simulations.

deposited on top of the stacks via thermal evaporation of $250 \mathrm{~nm}$ of copper and a protection layer of $5 \mathrm{~nm}$ of gold. A sinusoidal current is driven through the signal line of the coplanar waveguide, leading to an alternating magnetic field on the order of several tenths of a millitesla acting in the plane of the ferromagnetic elements. The provided in-plane magnetic contrast of the microscope yields the relative configuration of the circulations. Here, the sample is tilted by $60^{\circ}$ relative to the incident X-ray beam. As shown in the inset we observe both possible combinations $\left(c_{1} c_{2}=1\right.$ and $\left.c_{1} c_{2}=-1\right)$, indicating that the silicon spacer decreases interlayer exchange coupling between the vortices. For interlayer exchange-coupled vortices only one state would occur [33]. Stray fields at the side surfaces of the disks emerge when the vortices are deflected from their equilibrium position indicated in Fig. 1(b). In addition, the vortex core regions exhibit a coupling due to the out-of-plane component of the magnetization [Fig. 1(c)]. Both interaction effects are crucial to understand the collective behavior in a stack of vortices. Here, only the stray fields for equal circulations $\left(c_{1} c_{2}=1\right)$ of the vortices are depicted. They have been obtained from micromagnetic simulations of the investigated structures as described in the last part of this work.

\section{EXPERIMENTS}

In principle two coupled oscillators have two eigenmodes that describe all possible motions of the system. For stacks of magnetic vortices both modes have been observed in spin-torque oscillators [22]. In the experiments the dynamic behavior of a stack of vortices is investigated using a short magnetic field pulse ( $1 \mathrm{~ns}, 3 \mathrm{mT}$ ) pointing in the $y$ direction. The field pulse allows for the excitation of only one of the two modes [17]. The different circularities and polarities in the stacks then yield different frequencies and motions of the one excited mode. Due to symmetry considerations there are four possible nondegenerate states. All four combinations of the relative circulations and polarizations are imaged using out-of-plane magnetic contrast. Here, the sample is tilted by $90^{\circ}$ relative to the incident $\mathrm{x}$-ray beam. The trajectories of the core regions are shown in Fig. 2(a) and can be found as a movie in the Supplemental Material [34]. While for static magnetic fields the deflection depends only on the circularity, the initial deflection due to a nanosecond magnetic field pulse depends on the handedness $(c p= \pm 1)$ of the isolated magnetic vortex [35]. Subsequent to the initial deflection the vortex performs a damped gyration around the center of the disk, where the sense of gyration is determined by the polarity $p$ of the core. Isolated vortices with a positive polarity $(p=1)$ gyrate counterclockwise, while vortices with a negative core polarity gyrate $(p=-1)$ clockwise. The oscillation frequency of the isolated vortex is $240 \mathrm{MHz}$ for the investigated structures. In the stack the external field pulse individually deflects both vortices depending on their handedness. Thus, two identical vortex states $\left(p_{1} p_{2}=1, c_{1} c_{2}=1\right)$ are deflected in identical directions and gyrate on equal trajectories after the field pulse. Due to the additive contrast of the two structures, the different vortex cores cannot be distinguished in this case. Changing the circularity of one of the vortices results in opposite initial deflections yielding different trajectories of the vortex cores. The two vortex cores have the same sense of gyration. They gyrate around a common barycenter until they reach their equilibrium position and merge into a single black dot due to their direct superposition. This motion has also been described theoretically [23,31]. For both circularity combinations the frequency of gyration is approximately $410 \mathrm{MHz}$. It is comparable to the frequency of an isolated disk with the combined thickness of the stacked vortices. The frequencies have been obtained by the sum of the Fourier transforms of the two vortex core motions in the stack. They are depicted in Fig. 2(b). In the next step, the relative polarities of the vortex stacks are changed from equal $\left(p_{1} p_{2}=1\right)$ to opposite $\left(p_{1} p_{2}=-1\right)$ polarizations using self-organized state formation $[19,36,37]$. Thereby, the remaining nondegenerate states of opposite polarization are accessible. Two isolated vortices with opposite polarizations have a different sense of gyration that is also observed within the stack. The collective motions have a lower frequency of about $175 \mathrm{MHz}$ compared 

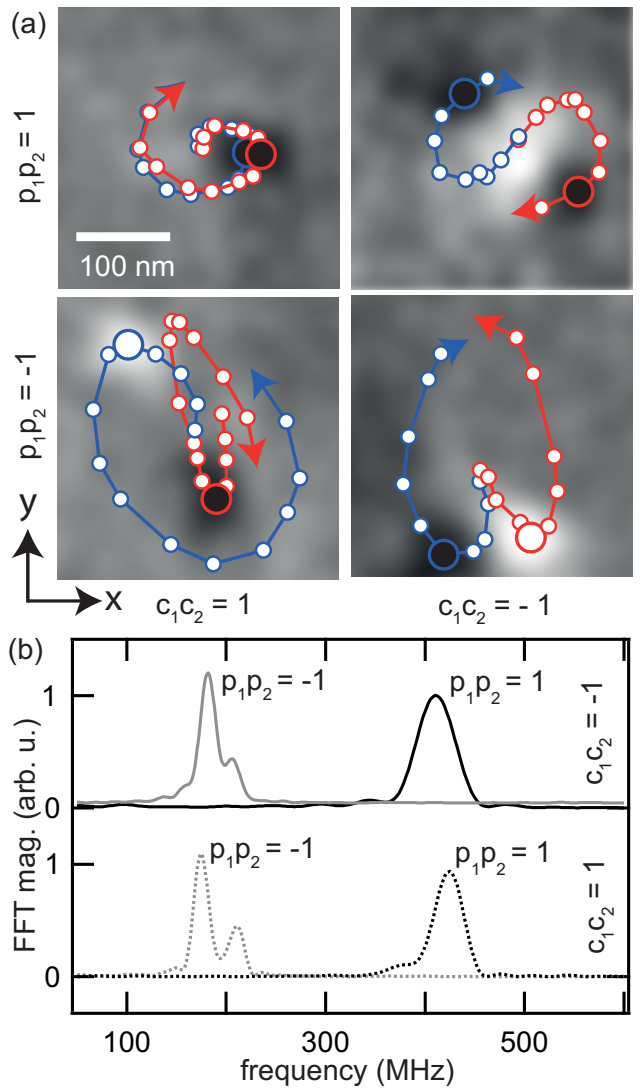

FIG. 2. (a) Collective trajectories of the vortex cores within the stack, indicated by red and blue lines. The out-of-plane component of each magnetic vortex is either black $(p=-1)$ or white $(p=1)$. It is obtained from the out-of-plane measurements. The vortices are excited by a short magnetic field pulse pointing in the $y$ direction. The motions depend on the indicated combination of the relative circulations and polarizations. (b) Sum of the Fourier transform of the two vortex core motions subsequent to a short magnetic field pulse. The relative circulations are indicated by dashed $\left(c_{1} c_{2}=1\right)$ and solid $\left(c_{1} c_{2}=-1\right)$ lines. The relative polarizations are depicted in black $\left(p_{1} p_{2}=1\right)$ and gray $\left(p_{1} p_{2}=-1\right)$.

to the resonance frequency of the isolated disks $(240 \mathrm{MHz})$. When the vortex cores approach each other, we observe a slight evasion that is attributed to the repulsion of the core regions. The two different relative polarities $\left(p_{1} p_{2}= \pm 1\right)$ have a strong influence on the resonances of the oscillations. The relative circulations yield slight variations. For the case of opposite polarities and equal circularities $\left(p_{1} p_{2}=-1\right.$, $\left.c_{1} c_{2}=1\right)$ a splitting of the resonances can be observed. It could be attributed to a change in the oscillation regime from large to small vortex core trajectories. This mode splitting can be observed for a critical core distance of about $50 \mathrm{~nm}$ in the movie (at $52 \mathrm{~ns}$ ) in the Supplemental Material [34]. Since the distance between the cores decreases over time, the influence of the core interaction increases. Further measurements of the steady-state motions elucidate this dependence.

The vortex stacks are excited near resonance by a sinusoidal magnetic field. Two different frequencies of 175 and $410 \mathrm{MHz}$ for the two relative polarizations $\left(p_{1} p_{2}= \pm 1\right)$ are used. Figure 3(a) illustrates the motions of the vortex cores for all
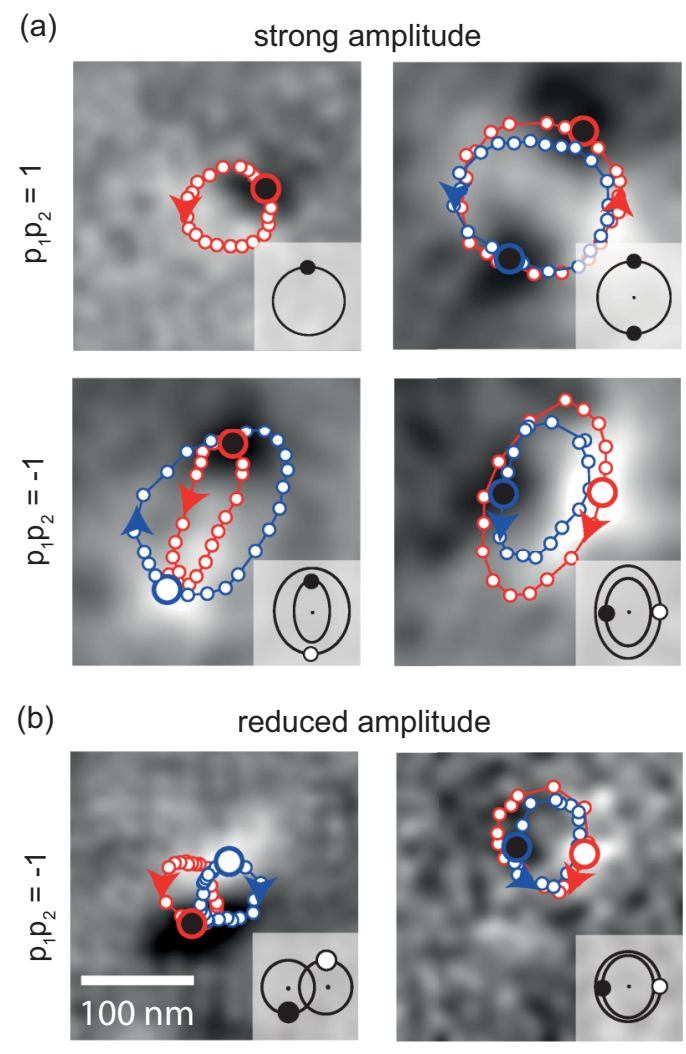

$\mathrm{c}_{1} \mathrm{c}_{2}=1$

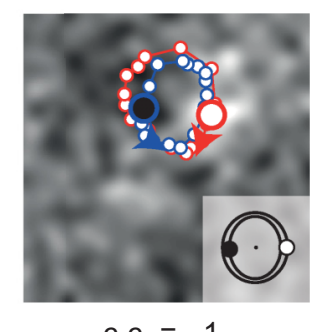

FIG. 3. (a) Stationary trajectories of all nondegenerate-state combinations in a stack of vortices, indicated by red and blue lines. The vortices are excited by a sinusoidal magnetic field pointing in the $y$ direction. Stacks with $p_{1} p_{2}=1$ are excited with a frequency of $410 \mathrm{MHz}$ and a field amplitude of $0.4 \mathrm{mT}$; a frequency of $175 \mathrm{MHz}$ is used for $p_{1} p_{2}=-1$ with an amplitude of $0.5 \mathrm{mT}$ for $c_{1} c_{2}=1$ and $0.8 \mathrm{mT}$ for $c_{1} c_{2}=-1$. (b) When the amplitude of the excitation is reduced by about $40 \%$ the type of the trajectory changes for one of the two relative circulations.

four state combinations. The vortices oscillate on constant trajectories that resemble possible motions of the gravitational two-body problem. Vortices with the same polarities gyrate on circular trajectories around a common barycenter. For equal circularities $\left(c_{1} c_{2}=1\right)$ the vortices gyrate on the same trajectory, whereas a phase shift of $180^{\circ}$ emerges for the case of opposite circularities $\left(c_{1} c_{2}=-1\right)$. In both cases the interaction is mediated by coupled in-plane dipoles rotating in the same direction. For equal circularities the attractive force of the two cores is zero since they gyrate on the same lateral positions. For opposite circularities the interaction of the cores can also be neglected. This is due to the large interdistance $(\sim 100 \mathrm{~nm})$ of the cores.

Vortices with opposite polarities gyrate in an opposite sense. Here, the relative phase and thereby the crossing point of the cores depend on the relative circulation. One of the cores is constrained to a lower radius. This asymmetry could be provoked by the Oersted field that slightly varies in its amplitude for the two disks. Then, the intrinsic repulsion of the cores strengthens this effect. However, as the oscillation radii are constant for the case of identical polarizations the asymmetry 
of the Oersted field is rather small. The amplitude of the excitation has been adjusted to maintain large-trajectory radii. By reducing the amplitude of the excitation the type of gyration changes for vortices with different polarities [Fig. 3(b)]. We observe a gyration of the vortices around individual barycenters for the case of equal circularities $\left(c_{1} c_{2}=1\right)$. Note that the barycenter is defined as the center of the core trajectory during one oscillation period of an individual disk. The vortices are repelled by the core interaction. This behavior cannot be observed for the case of opposite circularities $\left(c_{1} c_{2}=-1\right)$. Slight oscillations of the barycenters could not be observed due to the stroboscopic measurement method that integrates over millions of oscillation periods.

\section{THEORETICAL MODEL AND DISCUSSION}

The strong frequency splitting of the two polarity states can be understood within the model presented in Ref. [23]. Within this model the splitting between the two polarity states sums up to $310 \mathrm{MHz}$ for the investigated structures. This value is larger than the experimental splitting reported in Fig. 2, which can be explained by the overestimation of the rigid vortex approach [38]. Still, the observed repulsion of the vortex cores has to be taken into account $[26,31,32]$. Therefore, we performed calculations where the interaction of the vortex cores is modeled by an additional potential. We calculate the total energies of two deflected magnetic vortices using the Thiele approach [39], which considers the magnetic vortex as a rigid particle. Figure 4(a) depicts the stray fields of two magnetic dipoles, the vortex cores, in a stack of magnetic vortices. The energy of these dipoles is modeled by the assumption of two interacting point dipoles. It can be expressed as a function of their lateral deflection in opposite directions $\Delta r$. The amplitude of the dipole moment is derived from the actual size of the magnetic vortex core. Here, we assume that all magnetic moments in a cylindrical shape with a radius $r_{c}$ and the thickness $t$ of the disks point into the same out-of-plane direction. The energy is given by

$$
E_{\text {core }}=-\frac{\mu_{0} \pi M_{s}^{2} p_{1} p_{2} r_{c}^{4} t^{2}}{4\left[\Delta r^{2}+(t+\Delta z)^{2}\right]^{\frac{3}{2}}}\left(\frac{3(t+\Delta z)^{2}}{\Delta r^{2}+(t+\Delta z)^{2}}-1\right),
$$

where $(t+\Delta z)$ is the distance between the vertical centers of the two disks, $M_{s}$ is the saturation magnetization of permalloy, and $\Delta z$ is the thickness of the silicon spacer. The core interaction is repulsive for opposite $\left(p_{1} p_{2}=-1\right)$ and attractive for equal polarities $\left(p_{1} p_{2}=1\right)$. Reasonable values of $r_{c}=12 \mathrm{~nm} \mathrm{[6]} \mathrm{and} M_{s}=800 \mathrm{kA} / \mathrm{m}$ are assumed. The energy contribution of the confinement of the disks is modeled by a harmonic potential $[20,38]$ with a curvature $\kappa=1.72 \times 10^{-3} \mathrm{~kg} / \mathrm{s}^{2}$ and corresponds to a frequency of $\omega_{0} /(2 \pi)=240 \mathrm{MHz}$. For vortices the interaction of the stray fields at the side surfaces of the disks is also described by a harmonic potential when equally deflected in opposite directions. The coupling coefficient $\eta=1.45 \times 10^{-3} \mathrm{~kg} / \mathrm{s}^{2}$ is obtained in analogy to Ref. [10] from numerical integration of the magnetostatic energy between the side surfaces. The stray field energy depends on the relative circulations of the disks as depicted in Fig. 4(b). The sum of all energy contributions (a)

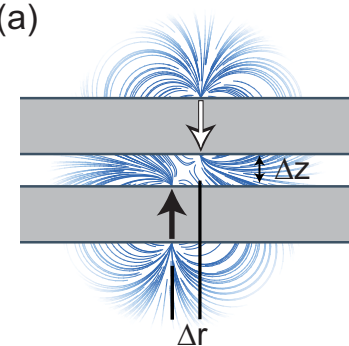

(c)

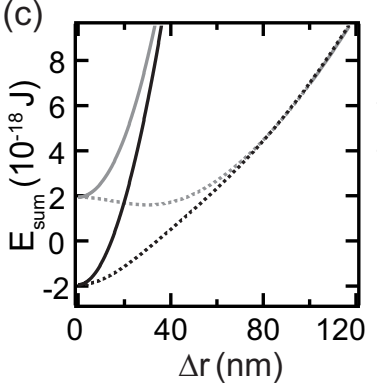

(b)
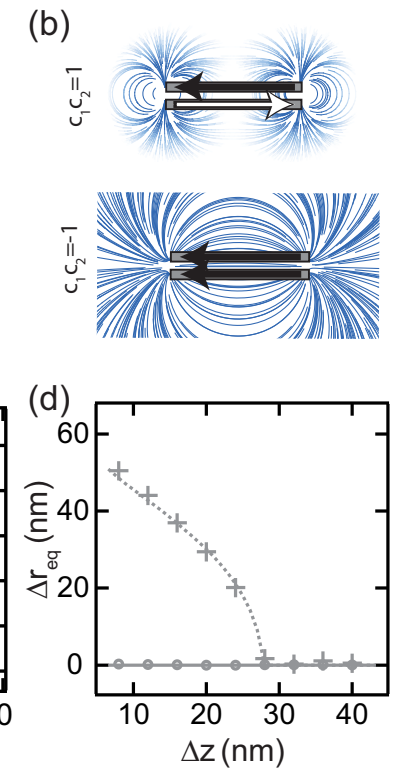

FIG. 4. Stray fields emerging (a) in the core regions and (b) at the side surfaces of vortices that are equally deflected in opposite directions. The stray fields have been calculated using dipoles indicated by black and white arrows. (c) Energy contributions as a function of the lateral distance of the vortex cores $\Delta r$ within the Thiele approach. All four nondegenerate combinations of the circulations (dashed: $c_{1} c_{2}=1$, solid: $c_{1} c_{2}=-1$ ) and the polarizations (black: $p_{1} p_{2}=1$, gray: $p_{1} p_{2}=-1$ ) are shown. (d) Resulting equilibrium position for opposite polarities as a function of the thickness $\Delta z$ of the silicon spacer. Micromagnetic simulations depicted by dots and crosses are in agreement with the calculations.

$E_{\text {sum }}$ reads

$$
E_{\text {sum }}=E_{\text {core }}\left(p_{1} p_{2}, \Delta r\right)+\left(\kappa-c_{1} c_{2} \eta\right) \frac{\Delta r^{2}}{4} .
$$

Figure 4(c) depicts the total energy $E_{\text {sum }}$ for all four possible nondegenerate state combinations. For large deflections $\Delta r$ the energy contribution of the stray fields at the side surfaces dominates. For the case of equal circularities and opposite polarities $\left(c_{1} c_{2}=1, p_{1} p_{2}=-1\right)$ the minimum energy, i.e., the equilibrium deflection $\Delta r_{e q}$, is obtained for $\Delta \mathrm{r}=30 \mathrm{~nm}$. All other state combinations reach their equilibrium position at $\Delta \mathrm{r}=0$. This behavior explains the collective oscillations observed in Fig. 3(b). Depending on the circularity, the two vortices oscillate around different or equal barycenters. For the case of equal circularities the distance between the two barycenters is approximately $\Delta \mathrm{r} \approx 50 \mathrm{~nm}$, whereas for the case of opposite circularities the two vortices gyrate around the same barycenter. These values are in good agreement with the analytical model. Slight variations can be explained by the measurement method that yields only the superposed contrast of both vortex cores. The energy between the vortex cores can also be calculated using the model of magnetic surface charges that emerge at the top and bottom surfaces of the disks. The proceeding is described in Ref. [26] and yields similar results. To gain insight into the strength of the observed dipolar coupling we performed further calculations to predict the limits of strong and weak core interactions. Figure 4(d) 
illustrates the dependence of the equilibrium deflection on the thickness of the silicon spacer. The analytical calculations are confirmed by micromagnetic simulations [40]. The dimensions of the simulated stacks are identical to the experiments. The equilibrium deflection is obtained by a relaxation of the system into its energetic minimum. Therefore, we use a cell size of $4 \times 4 \times 4 \mathrm{~nm}$, a saturation magnetization of $M_{s}=800 \mathrm{kA} / \mathrm{m}$, an exchange stiffness constant of $\mathrm{A}_{e x}=1.3 \times 10^{-11} \mathrm{~J} / \mathrm{m}$, and a Gilbert damping constant of $\alpha=0.01$. In agreement with Ref. [32] the case of equal circularities provokes a larger static displacement of the cores. Depending on the geometry of the disks, the displacement is expected to emerge for the case of different circularities as well.

\section{v. CONCLUSION}

We conclude that the collective magnetic excitation in a stack of vortices is dominated by the relative polarities of the vortex cores. A strongly increased splitting of the resonance frequencies compared to laterally coupled structures is observed. The proximity of the disks results in a coupling of the vortex cores. This coupling yields a displacement of the equilibrium positions for vortex stacks with equal circulations and opposite polarities. Its nonlinear influence leads to different types of steady-state motions observed by scanning transmission $\mathrm{x}$-ray microscopy that yield a two-body problem. The access to the third dimension in stacked vortices overcomes the limitations concerning the storage density in potential memory devices.

\section{ACKNOWLEDGMENTS}

We thank U. Merkt for fruitful discussions and M. Volkmann for superb technical assistance. We acknowledge the support of the Max Planck Institute for Intelligent Systems (formerly MPI for Metals Research), Department Schütz, and the MAXYMUS team, particularly, M. Bechtel and E. Goering. We thank the Helmholtz-Zentrum Berlin für Materialien und Energie for the allocation of synchrotron radiation beam time. Financial support of the Deutsche Forschungsgemeinschaft via the Sonderforschungsbereich 668 and the Graduiertenkolleg 1286 is gratefully acknowledged. This work has been supported by the excellence cluster "The Hamburg Centre for Ultrafast Imaging (CUI): Structure, Dynamics and Control of Matter at the Atomic Scale" of the Deutsche Forschungsgemeinschaft.
[1] V. V. Kruglyak, S. O. Demokritov, and D. Grundler, J. Phys. D 43, 264001 (2010).

[2] A. Hoffmann and S. D. Bader, Phys. Rev. Appl. 4, 047001 (2015).

[3] A. H. Eschenfelder, Magnetic Bubble Technology, Springer Series in Solid-State Sciences Vol. 14 (Springer, Berlin, 1981).

[4] S. S. P. Parkin, M. Hayashi, and L. Thomas, Science 320, 190 (2008).

[5] S. Mühlbauer, B. Binz, F. Jonietz, C. Pfleiderer, A. Rosch, A. Neubauer, R. Georgii, and P. Böni, Science 323, 915 (2009).

[6] A. Wachowiak, J. Wiebe, M. Bode, O. Pietzsch, M. Morgenstern, and R. Wiesendanger, Science 298, 577 (2002).

[7] T. Shinjo, T. Okuno, R. Hassdorf, K. Shigeto, and T. Ono, Science 289, 930 (2000).

[8] S. Bohlens, B. Krüger, A. Drews, M. Bolte, G. Meier, and D. Pfannkuche, Appl. Phys. Lett. 93, 142508 (2008).

[9] B. Van Waeyenberge, A. Puzic, H. Stoll, K. W. Chou, T. Tyliszczak, R. Hertel, M. Fähnle, H. Brück, K. Rott, G. Reiss, I. Neudecker, D. Weiss, C. H. Back, and G. Schütz, Nature (London) 444, 461 (2006).

[10] J. Shibata, K. Shigeto, and Y. C. Otani, Phys. Rev. B 67, 224404 (2003).

[11] A. Vogel, A. Drews, T. Kamionka, M. Bolte, and G. Meier, Phys. Rev. Lett. 105, 037201 (2010).

[12] J. Mejía-López, D. Altbir, A. H. Romero, X. Batlle, I. V. Roshchin, C.-P. Li, and I. K. Schuller, J. Appl. Phys. 100, 104319 (2006).

[13] S. Sugimoto, Y. Fukuma, S. Kasai, T. Kimura, A. Barman, and Y. C. Otani, Phys. Rev. Lett. 106, 197203 (2011).

[14] H. Jung, K.-S. Lee, D.-E. Jeong, Y.-S. Choi, Y.-S. Yu, D.-S. Han, A. Vogel, L. Bocklage, G. Meier, M.-Y. Im, P. Fischer, and S.-K. Kim, Sci. Rep. 1, 59 (2011).
[15] D.-S. Han, A. Vogel, H. Jung, K.-S. Lee, M. Weigand, H. Stoll, G. Schütz, P. Fischer, G. Meier, and S.-K. Kim, Sci. Rep. 3, 2262 (2013).

[16] J. Shibata and Y. C. Otani, Phys. Rev. B 70, 012404 (2004).

[17] M. Hänze, C. F. Adolff, M. Weigand, and G. Meier, Appl. Phys. Lett. 104, 182405 (2014).

[18] C. Behncke, M. Hänze, C. F. Adolff, M. Weigand, and G. Meier, Phys. Rev. B 91, 224417 (2015).

[19] M. Hänze, C. F. Adolff, M. Weigand, and G. Meier, Phys. Rev. B 91, 104428 (2015).

[20] B. Krüger, A. Drews, M. Bolte, U. Merkt, D. Pfannkuche, and G. Meier, Phys. Rev. B 76, 224426 (2007).

[21] Vortices have been observed in disks with a minimum diameter of $100 \mathrm{~nm}$. Thus, even for highest packing, storage densities of about $250 \mathrm{Gbit} / \mathrm{in}^{2}$ available in state-of-the-art hard disk drives cannot be achieved.

[22] R. Lebrun, N. Locatelli, S. Tsunegi, J. Grollier, V. Cros, F. Abreu Araujo, H. Kubota, K. Yakushiji, A. Fukushima, and S. Yuasa, Phys. Rev. Appl. 2, 061001 (2014).

[23] K. Y. Guslienko, K. S. Buchanan, S. D. Bader, and V. Novosad, Appl. Phys. Lett. 86, 223112 (2005).

[24] K. W. Chou, A. Puzic, H. Stoll, G. Schütz, B. Van Waeyenberge, T. Tyliszczak, K. Rott, G. Reiss, H. Brückl, I. Neudecker, D. Weiss, and C. H. Back, J. Appl. Phys. 99, 08F305 (2006).

[25] O. V. Sukhostavets, G. R. Aranda, and K. Y. Guslienko, J. Appl. Phys. 111, 093901 (2012).

[26] S. S. Cherepov, B. C. Koop, A. Yu. Galkin, R. S. Khymyn, B. A. Ivanov, D. C. Worledge, and V. Korenivski, Phys. Rev. Lett. 109, 097204 (2012).

[27] S. Wintz, C. Bunce, A. Neudert, M. Körner, T. Strache, M. Buhl, A. Erbe, S. Gemming, J. Raabe, C. Quitmann, and J. Fassbender, Phys. Rev. Lett. 110, 177201 (2013). 
[28] T. Tanigaki, Y. Takahashi, T. Shimakura, T. Akashi, R. Tsuneta, A. Sugawara, and D. Shindo, Nano Lett. 15, 1309 (2015).

[29] V. Sluka, A. Kákay, A. M. Deac, D. E. Bürgler, C. M. Schneider, and R. Hertel, Nat. Commun. 6, 6409 (2015).

[30] A. A. Awad, A. Lara, V. Metlushko, K. Y. Guslienko, and F. G. Aliev, Appl. Phys. Lett. 100, 262406 (2012).

[31] S.-H. Jun, J.-H. Shim, S.-K. Oh, S.-C. Yu, D.-H. Kim, B. Mesler, and P. Fischer, Appl. Phys. Lett. 95, 142509 (2009).

[32] F. Boust and N. Vukadinovic, IEEE Trans. Magn. 47, 349 (2011).

[33] S. Wintz, C. Bunce, A. Banholzer, M. Körner, T. Strache, R. Mattheis, J. McCord, J. Raabe, C. Quitmann, A. Erbe, and J. Fassbender, Phys. Rev. B 85, 224420 (2012).
[34] See Supplemental Material at http://link.aps.org/supplemental/ 10.1103/PhysRevB.93.054411 for a movie of the vortex core motions in the stacks.

[35] S.-B. Choe, Y. Acremann, A. Scholl, A. Bauer, A. Doran, J. Stöhr, and H. A. Padmore, Science 304, 420 (2004).

[36] C. F. Adolff, M. Hänze, A. Vogel, M. Weigand, M. Martens, and G. Meier, Phys. Rev. B 88, 224425 (2013).

[37] S. Jain, V. Novosad, F. Y. Fradin, J. E. Pearson, V. Tiberkevich, A. N. Slavin, and S. D. Bader, Nat. Commun. 3, 1330 (2012).

[38] K. Yu. Guslienko, B. A. Ivanov, V. Novosad, Y. C. Otani, H. Shima, and K. Fukamichi, J. Appl. Phys. 91, 8037 (2002).

[39] A. A. Thiele, Phys. Rev. Lett. 30, 230 (1973).

[40] MICROMAGNUM, http://micromagnum.informatik.unihamburg.de. 